\title{
ARTíCULO \\ Crecimiento y supervivencia del callo de hacha Atrina maura (Bivalvia: Pinnidae) cultivado en la costa sureste del Golfo de California, México
}

Growth and survival of pen shell Atrina maura (Bivalvia: Pinnidae) cultured in the southeastern coast of the Gulf of California, Mexico

\section{Andrés Martín Góngora-Gómez ${ }^{1}$, Manuel García-Ulloa ${ }^{1 *}$, Juan Antonio Hernández-Sepúlveda², Ana Laura Domínguez-Orozco ${ }^{2}$ and Juan Carlos Sainz-Hernández ${ }^{1}$}

\begin{abstract}
${ }^{1}$ Instituto Politécnico Nacional, Centro Interdisciplinario de Investigación para el Desarrollo Integral Regional-Unidad Sinaloa, Departamento de Acuacultura, Bulevar Juan de Dios Bátiz Paredes \#250, Colonia San Joachin, Guasave, Sinaloa, México. *turbotuag@ hotmail.com

${ }^{2}$ Instituto Politécnico Nacional, Centro Interdisciplinario de Investigación para el Desarrollo Integral Regional-Unidad Sinaloa, Departamento de Medio Ambiente, Bulevar Juan de Dios Bátiz Paredes \#250, Colonia San Joachin, Guasave, Sinaloa, México
\end{abstract}

\begin{abstract}
The evaluation of growth and survival of bivalves represents an important tool in culture. Growth performance and survival of the pen shell Atrina maura cultivated in Isla Los Redos, at the southeastern coast of the Gulf of California, Sinaloa, México, during 15 months (February 2008-May 2009), were described. The mean initial shell height and body weight of seeds ( $\mathrm{n}=$ 2,500 ) were $28.41 \pm 4.69 \mathrm{~mm}$ and $0.3 \pm 0.2 \mathrm{~g}$, respectively. Pen shells were cultured in suspension for 4 months, and 11 months in bottom system until harvesting. Biometrics of 50 specimens were measured every month. The average final shell height and weight were $220.48 \pm 12.41 \mathrm{~mm}$ and $284.26 \pm 54.45 \mathrm{~g}$, respectively. There were significant differences among the total weight of each month. The mean daily growth of shell height and body weight were $0.48 \mathrm{~mm} \mathrm{~d}^{-1}$ and $0.63 \mathrm{~g} \mathrm{~d}^{-1}$, respectively. Final survival was $88.72 \%$. Results show that A. maura reached the commercial size ( $\geq 200 \mathrm{~mm}$ shell height) after 9 months of culture in Isla Los Redos, Sinaloa, Mexico. This information contribute with basic knowledge for further studies on farming of the pen shell A. maura.
\end{abstract}

Key words: Pen shell production, growth rate, Gulf of California, bottom culture, management

Resumen.- El crecimiento y la supervivencia de bivalvos representan herramientas importantes para evaluar su cultivo. Se describe el crecimiento y la supervivencia del callo de hacha Atrina maura cultivado durante 15 meses (febrero 2008-mayo 2009) en Isla Los Redos, en la costa sureste del Golfo de California, México. El promedio inicial de la altura de la concha y el peso corporal de las semillas $(n=2500$ ) fueron $28,41 \pm 4,96 \mathrm{~mm}$ y $0,3 \pm 0,2 \mathrm{~g}$, respectivamente. Los organismos se mantuvieron 4 meses en suspensión y 11 meses sembrados en arena hasta la cosecha. Se realizaron biometrías de 50 especímenes cada mes. En promedio, los valores de crecimiento finales fueron de $220,48 \pm 12,41 \mathrm{~mm}$ para la altura de la concha y $284,26 \pm 54,45 \mathrm{~g}$ para el peso. Se encontraron diferencias significativas en el peso total durante la duración del cultivo. El promedio de la tasa diaria de crecimiento registró un valor de $0,48 \mathrm{~mm} \mathrm{~d}^{-1}$ y $0,63 \mathrm{~g} \mathrm{~d}^{-1}$ para la altura de la concha y el peso corporal, respectivamente. La supervivencia final fue de $88,72 \%$. Los resultados muestran que A. maura alcanzó la talla comercial $(\geq 200 \mathrm{~mm})$ después de 9 meses de cultivo en Isla Los Redos, Sinaloa, México. Esta información contribuye con conocimiento básico para posteriores estudios en el desarrollo del cultivo de A. maura.

Palabras clave: Producción de callo de hacha, tasa de crecimiento, Golfo de California, cultivo de fondo, manejo

\section{INTRODUCCIÓN}

El bivalvo Atrina maura (Sowerby, 1835) es conocido comúnmente en México como 'callo de hacha', nombre vulgar que se atribuye al atractivo comercial que representa la forma de su concha y su músculo aductor. Esta especie se distribuye desde ambos litorales de la península de Baja California, hasta Perú (Keen 1971), encontrándose en bahías protegidas de suelos arenosos, limosos o arcillosos (Coan \& Valentich-Scott 2012). Las poblaciones silvestres han disminuido al punto de su casi desaparición en las regiones costeras que habita debido a acciones antropogénicas, desastres naturales y al incremento desmedido de su captura (Camacho-Mondragón et al. 2012). De esta manera, su cultivo es una alternativa tanto para preservar el recurso como para incrementar su producción. Sin embargo, la información para producir semilla bajo condiciones de laboratorio es escasa (Robles et al. 1996, Serrano-Guzmán 1997, Robles-Mungaray 2004) y el aprovisionamiento de 
juveniles para su engorda comercial no es constante (MassóRojas et al. 1996, Singh-Cabanillas \& Michel-Guerrero 2002, Ángel-Dapa et al. 2015). La mayoría de la información relacionada a su engorda en México se ubica en los estados de Baja California, Baja California Sur (dentro y fuera del Golfo de California) y Sonora (Cardoza-Velasco 1998, Leal-Soto et al. 2011, Mendo et al. 2011). Recientemente, Góngora-Gómez et al. $(2011,2012)$ reportaron cultivos experimentales de $A$. maura en costas del estado de Sinaloa.

Tanto el crecimiento como la supervivencia representan indicadores determinantes en el cultivo del callo de hacha. Se ha concluido que estos factores pueden optimizarse con el manejo adecuado de la densidad de siembra (Góngora-Gómez et al. 2011), origen y manejo de los reproductores (ÁngelDapa et al. 2015), cantidad de nutrientes (Lora-Vilchis et al. 2004), elección del sitio de cultivo (Mendo et al. 2011), flujo de la corriente (Arrieche et al. 2010) y temperatura del agua (Leyva-Valencia et al. 2001, Rodríguez-Jaramillo et al. 2001), entre otros. Específicamente con pinnidos, Arizpe-Covarrubias (1995) reportó diferencias en el crecimiento y la mortalidad del callo de hacha Pinna rugosa cultivado en fondo y en suspensión, y Ahumada-Sempoal et al. (2002) evaluaron la abundancia y crecimiento de A. maura. También, SerranoGuzmán (2003) analizó las relaciones morfométricas de $P$. rugosa, mientras que Mendo et al. (2011) estudiaron el crecimiento, producción y mortalidad de $A$. maura en un cultivo de fondo intermareal; pero en los anteriores trabajos los organismos pertenecían a poblaciones naturales. Para el caso de juveniles de A. maura producidos en laboratorio, los trabajos acerca de su crecimiento y supervivencia se refieren a reportes con fines académicos y experimentales (MirandaBaeza 1994, Hernández 2006, Almaraz-Salas 2008, ÁngelDapa et al. 2015), y son pocos los relacionados a su producción comercial.

A pesar de que A. maura es una especie de importancia comercial y de alta demanda en el mercado, su cultivo en el estado de Sinaloa está basado en producciones esporádicas en el campo que no han permitido la generación de la información necesaria para implementar una biotecnología que optimice su producción. De esta manera, el objetivo del presente estudio fue la descripción del crecimiento y la supervivencia de A. maura cultivado en Isla Los Redos, Sinaloa, México, desde febrero 2008 a mayo 2009, y determinar la influencia de factores ambientales en su crecimiento.

\section{Materiales Y MÉTODOS}

\section{Área de ESTUdio}

El sitio de cultivo fue ubicado en la Isla Los Redos, localizado dentro del Sistema Lagunar Ensenada-Pabellones, perteneciente al municipio de Navolato, Sinaloa, México, en la costa sureste del Golfo de California (Fig. 1), entre los paralelos $24^{\circ} 30^{\prime} \mathrm{N}$ y $107^{\circ} 48^{\prime}$ O (SARH 1979).

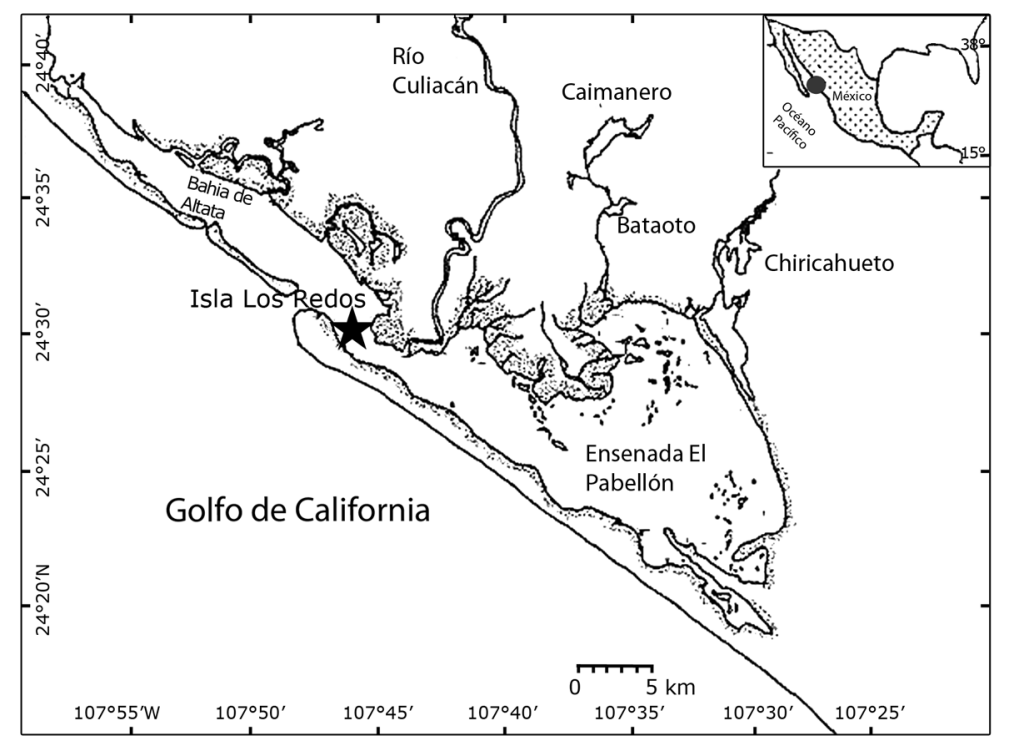

Figura 1. Ubicación del sitio de cultivo (Isla Los Redos) del callo de hacha A. maura, en el Sistema Lagunar Ensenada Pabellones, Sinaloa, México / Culture site (Isla Los Redos) of the pen shell A. maura, in the Ensenada Pabellones Lagoon System, Sinaloa, Mexico 


\section{Obtención de JuVeniles}

Se utilizaron 2500 semillas con una altura de la concha (distancia desde el umbo a la parte más distal de la concha) de 16,20 \pm $2,34 \mathrm{~mm}$ y con un peso promedio de $0,3 \pm 0,2 \mathrm{~g}$, obtenidas del Laboratorio del Instituto de Acuacultura de Sonora, perteneciente al Centro de Reproducción de Especies Marinas del Estado de Sonora, S. A. de C. V. (Bahía Kino, Sonora, México), las cuales fueron transportadas en seco y a baja temperatura $\left(\approx 20^{\circ} \mathrm{C}\right)$ al lugar de siembra.

\section{Cultivo}

El sistema de cultivo consistió en el uso de canastas ostrícolas durante los primeros 4 meses de engorda de las semillas (15 de febrero a 15 de junio), para después enterrarlas en fondo arenoso hasta el final del estudio (15 de junio 2008 a 15 de mayo 2009), conforme a la tecnología previa utilizada y reportada por Miranda-Baeza (1994) y Almaraz-Salas (2008). Los callos fueron aclimatados de acuerdo a Gallo-García et al. (2001) y repartidos en lotes dentro de bolsas de plástico mosquitero (2 $\mathrm{mm}$ de diámetro); posteriormente, las bolsas fueron colocadas en canastas ostrícolas para formar los módulos de cultivo que constaron de 2 canastas de protección y 5 de engorda; en estas últimas se ajustó una densidad inicial aproximada de 200 callos canasta $^{-1}$. Las canastas de protección se colocaron en la parte superior e inferior de cada módulo; la superior contenía un flotador el cual permitió al módulo mantenerse en suspensión. Una vez formados, los módulos fueron atados al sistema de cultivo de línea madre ó 'long-line' cuya cimentación consistió en 2 depósitos de lámina rellenos de cemento y grava -representando el peso muerto- para tensarlo y mantenerlo sujeto al fondo. A los extremos de la línea, se ató una cuerda con boyas que sirvieron como flotadores.

Los módulos o unidades de cultivo fueron limpiados cada 15 días para evitar la presencia de depredadores y/o epibiontes, y de esta manera, contabilizar los animales muertos. Una vez que los bivalvos alcanzaron la talla de $6 \mathrm{~mm}$ (15 de marzo 2008), fueron cultivados fuera de las bolsas, aún dentro de las canastas, hasta el 15 de mayo 2008. En total, los juveniles permanecieron 4 meses en las canastas para después, ser enterrados en el sustrato arenoso donde permanecieron 11 meses hasta la cosecha. Se construyó un corral o cerco con un área de $78 \mathrm{~m}^{2}$ (13 $\mathrm{m}$ de largo, $6 \mathrm{~m}$ de ancho y 1,70 $\mathrm{m}$ de altura) mediante un fardo de nylon de 3 pulgadas como límite físico, el cual fue enterrado $20 \mathrm{~cm}$ en la arena y expuesto $40 \mathrm{~cm}$ encima del nivel de la arena con el objetivo de proteger los animales de depredadores a manera de cerco y prevenir pérdidas; además, se utilizaron 3 postes de concreto a lo ancho y 5 a lo largo, a una distancia de 3,30 m entre ellos. Los corrales fueron construidos a una distancia de 10-20 m uno de otro. Los juveniles de A. maura fueron sembrados a una distancia mínima de $12-15 \mathrm{~cm}$ entre ellas, ajustando una densidad promedio de 36 callos $\mathrm{m}^{2}$.

\section{VARIABLES FISICOQUÍMICAS}

Se registraron las variables físicas y químicas al momento de la siembra y cada 15 días hasta el final del cultivo. La temperatura del agua y la concentración de oxígeno disuelto fueron obtenidos con un oxímetro (YSI, 55/12FT, Ohio 4587); en el caso de la salinidad, se utilizó un refractómetro de precisión (Atago, S/ Mill). El potencial de hidrógeno $(\mathrm{pH})$ se midió con un potenciómetro (Hanna, HI 8314). También, muestras de agua fueron tomadas cada mes y almacenadas en envases plásticos y transportadas en una hielera al Laboratorio de Análisis Ambiental del Instituto Politécnico Nacional (IPN-Unidad Sinaloa) para su posterior análisis. Se determinó amonio $\left(\mathrm{NH}_{3}\right)$ con la técnica descrita por Solórzano (1969) a $640 \mathrm{~nm}$ de absorbancia; nitritos $\left(\mathrm{NO}_{2}\right)$ por medio del método de Shinn (1941) a $543 \mathrm{~nm}$ de absorbancia y aplicado al agua de mar (Bendschneider \& Robinson 1952); y fosfatos $\left(\mathrm{PO}_{4}\right)$ utilizando la metodología de Murphy \& Riley (1962) a 420 nm de absorbancia. Todas las lecturas de los parámetros se realizaron con un espectrofotómetro Termo Spectronic Genesys 2. Las muestras de agua para determinar clorofila $a(\mathrm{Cl} a)$ fueron filtradas con filtros de fibra de vidrio Whatman $(\mathrm{GF} / \mathrm{F} 0,7 \mu \mathrm{m})$, los cuales se congelaron a $-20^{\circ} \mathrm{C}$ hasta efectuar la extracción del pigmento con acetona al $90 \%$ durante 24 h, según lo recomendado por Strickland \& Parsons (1972). Los cálculos de la concentración de $\mathrm{Cl} a$ se realizaron de acuerdo a las ecuaciones de Jeffrey \& Humphrey (1975).

\section{Crecimiento y Supervivencia}

Se midieron in situ 50 organismos cada mes. Las biometrías se realizaron con un Vernier digital (Mitutoyo, CD-8" CS, 0,01 $\mathrm{mm}$ de precisión) para determinar la altura de la concha (distancia desde el umbo hasta la parte más distal de la concha). Se utilizó una balanza granataria (OHAUS, Scout Pro SP 2001; 0,01 g de precisión) para la obtención del peso húmedo total de cada organismo, previo secado con papel absorbente.

Para obtener los valores mensuales de la altura de la concha y el peso ganados por día, se calcularon la tasa diaria de crecimiento $(\mathrm{TCD}), \mathrm{TCD}=\left(L_{2}-L_{1} / \mathrm{t}\right)$, y la tasa de crecimiento absoluto (TCA), TCA $=\left(\mathrm{Y}_{2}-\mathrm{Y}_{1}\right) /\left(\mathrm{t}_{2}-\mathrm{t}_{1}\right)$, donde $\mathrm{Y}_{2}$ y $\mathrm{Y}_{1}=$ peso $o$ altura de la concha final e inicial, respectivamente, $t_{2} y$ $\mathrm{t}_{1}=$ tiempo final e inicial de cultivo entre dos muestreos (Wootton 1991). 
La supervivencia fue determinada por la resta del número de conchas vacías recolectadas de los muestreos mensuales del total de la población inicial, hasta el término del estudio.

\section{Análisis de DATOS}

Para analizar los datos de crecimiento se aplicó un ANOVA de una vía, empleando como factor el tiempo de cultivo. Cuando se detectaron diferencias significativas, se aplicó una prueba de Tukey $(P<0,05)$. Para determinar el grado de asociación del crecimiento y la supervivencia de los callos con los parámetros fisicoquímicos, se realizó una correlación de Pearson (r) a una significancia de 95\%. Los datos de supervivencia expresados en porcentaje fueron transformados a arcoseno para ser normalizados (Zar 1996). También se obtuvo la correlación entre la temperatura del agua y la cantidad de Cla por ser los parámetros más importantes en el crecimiento y supervivencia de bivalvos (Newell 1976, Leal-Soto et al. 2011), mismos que fueron correlacionados con las tasas de crecimiento. Todos los análisis estadísticos fueron realizados con el programa Statgraphic Plus, ver. 5 (Statistical Graphics Corp., Herndon, VA, USA).

\section{Resultados}

\section{VARIABLES FISICOQUíMICAS}

La temperatura del agua registrada en Isla Los Redos fluctuó desde $21,2^{\circ} \mathrm{C}$ en marzo 2008 y enero 2009 , hasta $31,4^{\circ} \mathrm{C}$ en marzo 2009. La salinidad mostró el valor máximo en marzo 2008 (36), y el mínimo en junio 2009 (31). La mayor concentración de Cla $\left(8,51 \mathrm{mg} \mathrm{m}^{-3}\right)$ fue obtenida en octubre 2008 , mientras que el menor valor $\left(2,92 \mathrm{mg} \mathrm{m}^{-3}\right)$ se observó en junio 2009, con un promedio de $5,25 \mathrm{mg} \mathrm{m}^{-3}$ (Fig. 2).

La concentración del oxígeno disuelto (OD) registró un promedio de 5,9 $\pm 1.2 \mathrm{mg} \mathrm{L}^{-1}$. El valor máximo $\left(7,6 \mathrm{mg} \mathrm{L}^{-1}\right) \mathrm{se}$ observó en febrero 2008 y el mínimo $\left(5,1 \mathrm{mg} \mathrm{L}^{-1}\right)$ en octubre 2008 . En cuanto al pH, el máximo valor $(8,6)$ se observó en varios meses de ambos años, mientras que el mínimo $(7,7)$ se encontró en marzo 2009 (Fig. 3).

El promedio en la concentración de $\mathrm{NH}_{3}$ fue de $0,98 \pm 0,16$ $\mathrm{mg} \mathrm{L}^{-1}$. La mayor concentración $\left(2,16 \mathrm{mg} \mathrm{L}^{-1}\right)$ se registró en abril 2008, mientras que la mínima $\left(0,05 \mathrm{mg} \mathrm{L}^{-1}\right)$ fue encontrada en mayo 2009. Para la concentración de $\mathrm{NO}_{2}$, el valor promedio fue de $0,16 \pm 0,04 \mathrm{mg} \mathrm{L}^{-1}$. La máxima concentración $(0,41 \mathrm{mg}$ $\left.\mathrm{L}^{-1}\right)$ se observó en febrero 2008, mientras que la mínima $(0,02$ $\mathrm{mg} \mathrm{L^{-1 }}$ ) se encontró en mayo 2009. En el caso de $\mathrm{PO}_{4}$, la concentración promedio fue de 1,39 $\pm 0,31 \mathrm{mg} \mathrm{L}^{-1}$, registrando la mayor concentración $\left(2,25 \mathrm{mg} \mathrm{L}^{-1}\right)$ en febrero 2008, mientras que la menor $\left(0,65 \mathrm{mg} \mathrm{L}^{-1}\right)$ se presentó en febrero 2009 (Fig. 4). La temperatura estuvo inversamente correlacionada con la salinidad $(\mathrm{r}=-0,67), \mathrm{OD}(\mathrm{r}=-0,76), \mathrm{NH}_{3}(\mathrm{r}=-0,58)$ y $\mathrm{NO}_{2}$ $(r=-0,57)$. Se obtuvo una baja correlación $(r=0,29)$ entre la temperatura del agua y la concentración de $\mathrm{Cl} a$ (Tabla 1).

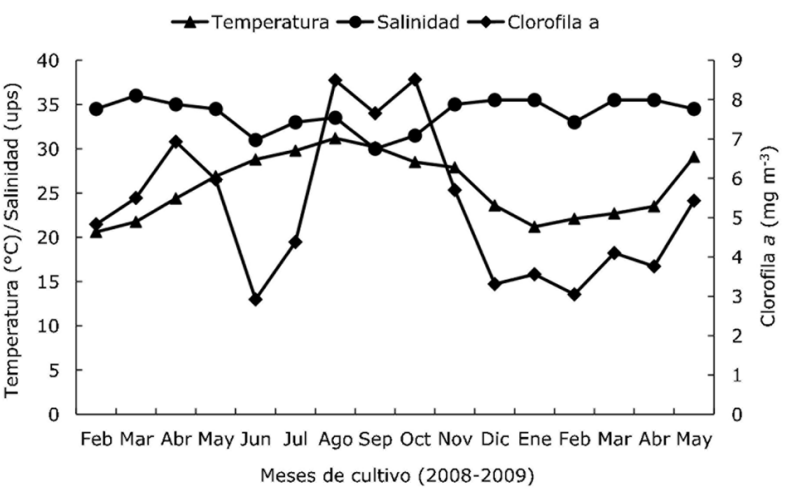

Figura 2. Variación de la clorofila a $\left(\mathrm{mg} \mathrm{m}^{-3}\right)$, temperatura $\left({ }^{\circ} \mathrm{C}\right) \mathrm{y}$ salinidad registradas desde febrero 2008 a mayo 2009, en Isla Los Redos, Sinaloa, México / Variation of chlorophyll a $\left(\mathrm{mg} \mathrm{m}^{-3}\right)$, temperature $\left({ }^{\circ} \mathrm{C}\right.$ ) and salinity registered from February 2008 to May 2009 in Isla Los Redos, Sinaloa, Mexico

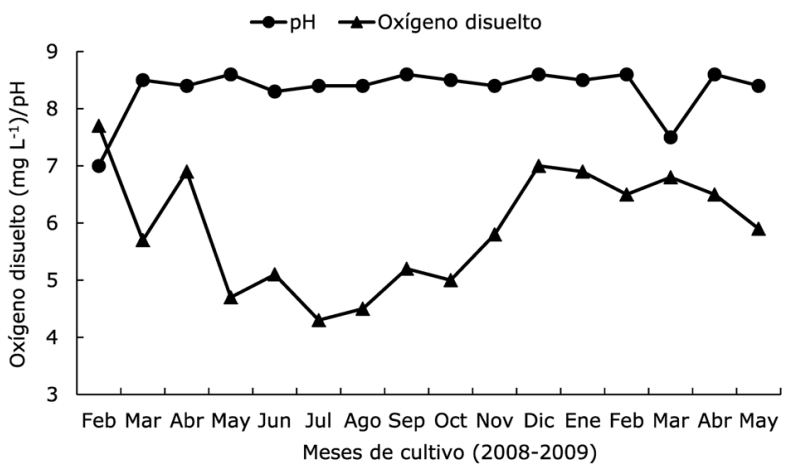

Figura 3. Variaciones de oxígeno disuelto $\left(\mathrm{mg} \mathrm{L}^{-1}\right)$ y $\mathrm{pH}$, registradas desde febrero 2008 a mayo 2009, en Isla Los Redos, Sinaloa, M éxico / Variations of dissolved oxygen ( $\mathrm{mg} \mathrm{L}^{-1}$ ) and $\mathrm{pH}$ registered from February 2008 to May 2009 in Isla Los Redos, Sinaloa, Mexico

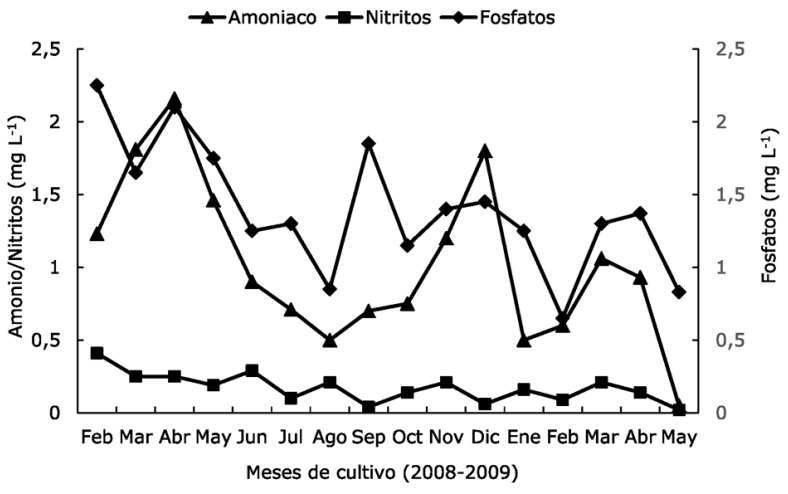

Figura 4. Variación de fosfatos $\left(\mathrm{PO}_{4}, \mathrm{mg} \mathrm{L}^{-1}\right)$, amonio $\left(\mathrm{NH}_{3}, \mathrm{mg} \mathrm{L}^{-1}\right)$ y nitritos $\left(\mathrm{NO}_{2}, \mathrm{mg} \mathrm{L}^{-1}\right)$ registradas desde febrero 2008 a mayo 2009, en Isla Los Redos, Sinaloa, México / Variation of phosphates $\left(\mathrm{PO}_{4}, \mathrm{mg}\right.$ $\left.\mathrm{L}^{-1}\right)$, ammonium $\left(\mathrm{NH}_{3}, \mathrm{mg} \mathrm{L}^{-1}\right)$ and nitrites $\left(\mathrm{NO}_{2}, \mathrm{mg} \mathrm{L}^{-1}\right)$ registered from February 2008 to May 2009, in Isla Los Redos, Sinaloa, Mexico 
Tabla 1. Correlaciones ( $r$ ) de los parámetros físicos, químicos y biológicos del agua, altura de la concha, peso corporal y supervivencia de A. maura cultivado en Isla Los Redos, Sinaloa, México / Correlations ( $r$ ) of physical, chemical and biological parameters, shell height, body weight and survival of A. maura cultured in Isla Los Redos, Sinaloa, Mexico

\begin{tabular}{|c|c|c|c|c|c|c|c|c|c|c|c|}
\hline & $\mathrm{AC}^{*}$ & $\mathrm{PC}$ & SP & $\mathrm{T}^{\circ} \mathrm{C}$ & UPS & OD & $\mathrm{pH}$ & $\mathrm{NH}_{3}$ & $\mathrm{NO}_{2}$ & $\mathrm{PO}_{4}$ & $\mathrm{Cl} a$ \\
\hline $\mathrm{AC}$ & & $0,98^{\S}$ & 0,92 & & & & & $-0,54$ & $-0,77$ & $-0,72$ & \\
\hline PC & & & 0,97 & & & & & $-0,51$ & $-0,66$ & $-0,67$ & \\
\hline \multicolumn{12}{|l|}{ SP } \\
\hline $\mathrm{T}^{\circ} \mathrm{C}$ & & & & & $-0,67$ & $-0,76$ & & $-0,58$ & $-0,57$ & & \\
\hline UPS & & & & & & 0,54 & & & & & \\
\hline \multicolumn{12}{|l|}{ OD } \\
\hline pH & & & & & & & & & $-0,62$ & & \\
\hline $\mathrm{NH}_{3}$ & & & & & & & & & 0,50 & 0,67 & \\
\hline $\mathrm{NO}_{2}$ & & & & & & & & & & 0,59 & \\
\hline $\mathrm{PO}_{4}$ & & & & & & & & & & & \\
\hline $\mathrm{Cl} a$ & & & & & & & & & & & \\
\hline
\end{tabular}

*AC $=$ altura de la concha $(\mathrm{mm}), \mathrm{PC}=$ peso corporal $(\mathrm{g}), \mathrm{SP}=$ supervivencia $(\%), \mathrm{T}^{\circ} \mathrm{C}=$ temperatura, $\mathrm{UPS}=$ salinidad, $\mathrm{OD}=$ oxígeno disuelto $\left(\mathrm{mg} \mathrm{L}^{-1}\right), \mathrm{NH}_{3}=$ amonio $\left(\mathrm{mg} \mathrm{L}^{-1}\right), \mathrm{NO}_{2}=$ nitritos $\left(\mathrm{mg} \mathrm{L}^{-1}\right)$, $\mathrm{PO}_{4}=$ fosfato $\left(\mathrm{mg} \mathrm{L}^{-1}\right), \mathrm{Cl} a=$ clorofila $a\left(\mathrm{mg} \mathrm{m}^{-3}\right)$

${ }^{\S} \mathrm{Se}$ indican solamente las correlaciones con significancia a una probabilidad de $95 \%$

\section{Crecimiento}

El análisis de varianza mostró diferencias significativas $(P>$ $0,05)$ en la altura de la concha $(\mathrm{F}=1290,87 ; P=0,0001)$ y el peso $(\mathrm{F}=614,43 ; P=0,0001)$ de $A$. maura durante los 15 meses de cultivo. Para la altura de la concha, se obtuvo un promedio final de 220,48 $\pm 12,41 \mathrm{~mm}$ (Tabla 2). El valor máximo para TCA $\left(1,12 \mathrm{~mm} \mathrm{~d}^{-1}\right)$ se presentó en el primer mes (febrero-marzo 2008), mientras que el mínimo correspondió en abril 2009 (-0,34 $\left.\mathrm{mm} \mathrm{d}^{-1}\right)$. El análisis de correlación mostró que la altura de la concha se relacionó positivamente con el peso $(r=0,98)$ y sin significancia con la Cla $(r=0,02)$.

Con respecto al peso total (Tabla 3), se observó un aumento progresivo desde $0,3 \pm 0,16 \mathrm{~g}$ para la población inicial, hasta $284,26 \pm 54,45 \mathrm{~g}$ al final del cultivo. El valor máximo de TCA se registró en abril-mayo $2009\left(\mathrm{TCA}=2,36 \mathrm{~g} \mathrm{~d}^{-1}\right)$, mientras que el mínimo en marzo-abril $2009\left(\mathrm{TCA}=-0,2103 \mathrm{~g} \mathrm{~d}^{-1}\right)$. Se obtuvo una correlación negativa e inversa entre el peso corporal y $\mathrm{Cl} a(\mathrm{r}=-0,24)$. El promedio de la tasa diaria de crecimiento registró un valor de $0,48 \mathrm{~mm} \mathrm{~d}^{-1}$ y $0,63 \mathrm{~g} \mathrm{~d}^{-1}$ para la altura de la concha y el peso corporal, respectivamente. Los nutrientes

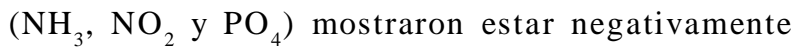
correlacionados con la altura de la concha y el peso corporal (Tabla 1).

La supervivencia final fue de 88,72\% (Fig. 5). Al correlacionar la supervivencia con la altura de la concha $(\mathrm{r}=$ $0,92)$ y el peso total $(r=-0,97)$, se obtuvieron relaciones inversas significativas, mientras que la supervivencia con la $\mathrm{Cl} a(\mathrm{r}=0,32)$, la temperatura $(\mathrm{r}=0,09)$ y la salinidad $(\mathrm{r}=0,22)$, mostraron baja correlación.
Tabla 2. Altura ( $\mathrm{mm}$ ) y tasa de crecimiento absoluto (TCA, $\mathrm{mm} \mathrm{d}^{-1}$ ) de la concha de A. maura cultivada en Isla Los Redos, Sinaloa, México / Shell height $(\mathrm{mm})$ and absolute growth rate (TCA, $\mathrm{mm} \mathrm{d}^{-1}$ ) of A. maura cultured in Isla Los Redos, Sinaloa, Mexico

\begin{tabular}{lrr}
\hline \multicolumn{1}{c}{ Fecha } & \multicolumn{1}{c}{$\begin{array}{c}\text { Altura de la } \\
\text { concha }(\mathrm{mm})\end{array}$} & $\begin{array}{r}\text { TCA } \\
\left(\mathrm{mm} \mathrm{d}^{-1}\right)\end{array}$ \\
\hline Feb. 2008 & $16,20 \pm 2,34^{\mathrm{a}^{\mathrm{*}}}$ & \\
Marzo & $51,00 \pm 1,92^{\mathrm{b}}$ & 1,12 \\
Abril & $82,62 \pm 7,35^{\mathrm{c}}$ & 1,02 \\
Mayo & $93,24 \pm 6,95^{\mathrm{cd}}$ & 0,34 \\
Junio & $119,66 \pm 8,98^{\mathrm{d}}$ & 0,88 \\
Julio & $145,13 \pm 9,03^{\mathrm{e}}$ & 0,85 \\
Agosto & $157,15 \pm 15,62^{\mathrm{e}}$ & 0,40 \\
Septiembre & $164,48 \pm 10,23^{\mathrm{ef}}$ & 0,23 \\
Octubre & $182,76 \pm 7,89^{\mathrm{ef}}$ & 0,61 \\
Noviembre & $207,15 \pm 11,02^{\mathrm{f}}$ & 0,81 \\
Diciembre & $206,14 \pm 10,63^{\mathrm{f}}$ & $-0,03$ \\
Ene. 2009 & $209,62 \pm 20,45^{\mathrm{f}}$ & 0,11 \\
Febrero & $206,72 \pm 9,61^{\mathrm{f}}$ & $-0,09$ \\
Marzo & $218,16 \pm 11,57^{\mathrm{f}}$ & 0,38 \\
Abril & $207,68 \pm 8,37^{\mathrm{f}}$ & $-0,34$ \\
Mayo & $220,48 \pm 10,64^{\mathrm{f}}$ & 0,42 \\
\hline
\end{tabular}

*En la columna, valores con diferente letra son estadísticamente diferentes $(P<0,05)$ 
Tabla 3. Peso corporal (g) y tasa de crecimiento absoluto (TCA $\mathrm{g} \mathrm{d}^{-1}$ ) de A. maura cultivada en Isla Los Redos, Sinaloa, México / Body weight (g) and absolute growth rate (TCA $\mathrm{g} \mathrm{d}^{-1}$ ) of A. maura cultured in Isla Los Redos, Sinaloa, Mexico

\begin{tabular}{lrc}
\hline Fecha & \multicolumn{1}{c}{$\begin{array}{c}\text { Peso } \\
(\mathrm{g})\end{array}$} & $\begin{array}{l}\text { TCA } \\
\left(\mathrm{g} \mathrm{d}^{-1}\right)\end{array}$ \\
\hline Feb. 2008 & $0,3 \pm 0,16^{\mathrm{a} *}$ & \\
Marzo & $2,03 \pm 0,61^{\mathrm{b}}$ & 0,057 \\
Abril & $11,40 \pm 2,98^{\mathrm{c}}$ & 0,312 \\
Mayo & $17,47 \pm 5,37^{\mathrm{c}}$ & 0,202 \\
Junio & $38,68 \pm 10,34^{\mathrm{d}}$ & 0,707 \\
Julio & $73,88 \pm 20,59^{\mathrm{e}}$ & 1,173 \\
Agosto & $83,99 \pm 19,04^{\mathrm{e}}$ & 0,337 \\
Septiembre & $98,63 \pm 26,46^{\mathrm{e}}$ & 0,488 \\
Octubre & $143,62 \pm 28,75^{\mathrm{f}}$ & 1,499 \\
Noviembre & $194,19 \pm 28,38^{\mathrm{fg}}$ & 1,685 \\
Diciembre & $198,19 \pm 35,20^{\mathrm{g}}$ & 0,133 \\
Ene. 2009 & $195,55 \pm 31,66^{\mathrm{g}}$ & $-0,088$ \\
Febrero & $213,99 \pm 43,32^{\mathrm{g}}$ & 0,614 \\
Marzo & $219,72 \pm 19,97^{\mathrm{g}}$ & 0,191 \\
Abril & $213,41 \pm 28,34^{\mathrm{g}}$ & $-0,210$ \\
Mayo & $284,26 \pm 54,45^{\mathrm{h}}$ & 2,361 \\
\hline
\end{tabular}

*En la columna, valores con diferente letra son estadísticamente diferentes $(P<0,05)$

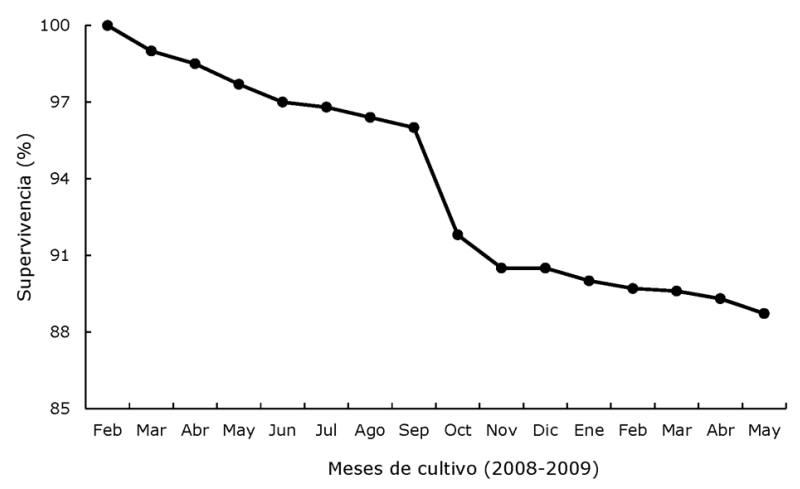

Figura 5. Supervivencia (\%) de A. maura cultivada en Isla Los Redos, Sinaloa, México / Survival (\%) of A. maura cultured in Isla Los Redos, Sinaloa, Mexico

\section{Discusión}

Las diferencias en las tasas de crecimiento de bivalvos reportadas en diversos trabajos reflejan la interacción de algunos factores ambientales (Wallace \& Reinsnes 1985, Rupp et al. 2005), principalmente la temperatura y Cla. Los resultados obtenidos en el presente trabajo mostraron diferencias significativas en los valores de la altura de la concha y peso de A. maura considerando el tiempo de cultivo como variable independiente. La altura de la concha alcanzó un promedio de $220,48 \pm 12,41 \mathrm{~mm}$ y el peso de $284,26 \pm 54,45 \mathrm{~g}$, después de 15 meses de cultivo. El crecimiento de la altura de la concha registrado $\left(0,45 \mathrm{~mm} \mathrm{~d}^{-1}\right)$ es comparable con los reportados por el cultivo de fondo $\left(0,40 \mathrm{~mm} \mathrm{~d}^{-1}\right)$ de Arizpe-Covarrubias (1995) y el cultivo combinado (suspensión y siembra en arena, 0,40 $\mathrm{mm} \mathrm{d}^{-1}$ ) de Miranda-Baeza (1995), pero mayor al obtenido por Cardoza-Velasco \& Maeda-Martínez (1997) y Leal-Soto et al. (2011) quienes en cultivo combinado reportaron 0,36 y $0,27 \mathrm{~mm} \mathrm{~d}^{-1}$, respectivamente, para la misma especie. Las diferencias pudieran explicarse por las condiciones ambientales de cada localidad y las variaciones en el sistema de cultivo de cada trabajo. A pesar del intervalo de temperatura registrado $\left(10,2^{\circ} \mathrm{C}\right.$ entre la máxima y la mínima), este parámetro no mostró relación significativa con la TCA de la altura de la concha $(\mathrm{r}=-0,43)$ y el peso $(\mathrm{r}=-0,45)$, lo que sugiere que la temperatura no afectó el crecimiento de A. maura. La temperatura del agua mostró un patrón normal de aparición con picos de valores máximos en mayo/junio y la disminución menor en diciembre/enero, lo cual coincide con los cambios estacionales de la zona.

Las concentraciones medias de los nutrientes muestreados $\left(\mathrm{NH}_{3}, \mathrm{NO}_{2}\right.$ y $\left.\mathrm{PO}_{4}\right)$ no fueron limitantes para el crecimiento poblacional de las comunidades fitoplanctónicas (Langton et al. 1977, Menzel 1991). En el caso de la disponibilidad de alimento, representada por la concentración de Cla, no mostró una relación significativa con la altura de la concha $(r=-0,02)$ y el peso $(r=0,24)$ de los bivalvos, lo que sugiere que los animales no dependieron directamente del fitoplancton para alimentarse, sino de otras fuentes como la materia orgánica particulada (MOP) encontrada en el seston, característica de hábitat de fondos lodosos como los reportados por Sibaja (1985), ArizpeCovarrubias (1995) y Almaraz-Salas (2008).

La supervivencia de A. maura se mantuvo por encima de $90 \%$ hasta el mes 13 de cultivo, para después disminuir hasta $88,72 \%$, la cual puede considerarse como aceptable para organismos en cultivo (Bunting 2013). Los factores que pueden afectar la supervivencia en cultivos de moluscos pueden ser la presencia de epibiontes, depredadores, competidores por espacio y alimento, así como efecto de corrientes (SerranoCasillas 2004), cambio brusco de parámetros (Acarli et al. 
2011) y manejo (Gallo-García et al. 2001). En este trabajo, la temperatura y disponibilidad de alimento no fueron factores significativos en la mortalidad de A. maura, ya que no existió asociación entre dichos parámetros con la mortalidad, además de que el crecimiento de los bivalvos fue sostenido hasta el mes 10 de cultivo. La disminución en el porcentaje de supervivencia, pudo haberse debido a organismos epibiontes y depredadores (Alcaráz-Zamora \& Mendo-Aguilar 2008). De acuerdo a Wu \& Shin (1998), la mortalidad en especies de bivalvos que se fijan al sustrato puede ser causada por: pérdida entre muestreos, depredación por peces, cangrejos y caracoles, y por causas desconocidas, cuando las conchas son colectadas vacías. Durante los muestreos, se observaron algunos organismos epífitos o competidores como balanos, algas, moluscos, crustáceos, peces y jaibas.

Los resultados obtenidos en el cultivo de A. maura en Isla Los Redos, Sinaloa, confirman su potencial acuícola en la zona, y asimismo, aportan información básica para futuros estudios de esta especie en la región. De acuerdo a la demanda de los vendedores locales, este bivalvo alcanzó la talla comercial (> $200 \mathrm{~mm}$ ) en noviembre 2008, es decir, 9 meses después de haber sido sembrado.

\section{Agradecimientos}

Los autores agradecen al Instituto Politécnico Nacional (IPN) el apoyo logístico y económico por medio de la Comisión de Operaciones y Fomento de Actividades Académicas (COFAA) al proyecto, IPN-SIP 20100618 (Efecto de la densidad de siembra en el cultivo de callo de hacha Atrina maura en la ensenada La Palmita, Navolato, Sinaloa).

\section{LITERATURA CITADA}

Acarli S, A Lok, A Kdermenc, H Yildiz \& S Serdar. 2011. Comparative growth, survival and condition index of flat oyster, Ostrea edulis (Linnaeus 1758) in Mersin Bay, Aegean Sea, Turkey. Kafkas University of Veterinary Fak Derg 17: 203-210.

Ahumada-Sempoal MA, SJ Serrano-Guzmán \& N RuízGarcía. 2002. Abundancia, estructura poblacional y crecimiento de Atrina maura (Bivalvia: Pinnidae) en la laguna costera tropical del Pacífico mexicano. Revista de Biología Tropical 50: 1091-1100.

Alcaráz-Zamora JA \& J Mendo-Aguilar. 2008. Crecimiento y supervivencia de juveniles de Argopecten purpuratus en sistemas de fondo y suspendido en la zona de Casma, Perú. Ecología Aplicada 7(1-2): 71-80.

Almaraz-Salas JC. 2008. Primer cultivo experimental de callo de hacha Atrina maura (Sowerby, 1835) en la ensenada La Palmita, Navolato, Sinaloa. Tesis de Licenciatura, Universidad de Occidente, Unidad Guasave, Guasave, Sinaloa, 74 pp.
Ángel-Dapa MA, M Arellano-Martínez, BP CeballosVázquez, M Robles-Mungaray, E Robles-Rocha, MA Camacho-Mondragón \& PE Saucedo. 2015. Reproductive and larval performance of the pen shell Atrina maura in relation to the origin and condition of broodstock. Journal of Shellfish Research 34: 401-408.

Arizpe-Covarrubias O. 1995. Mortality, growth and somatic secondary production of the bivalve, Pinna rugosa (Sowerby), in suspended and bottom culture in Bahia de La Paz, México. Aquaculture Research 26: 843-853.

Arrieche D, AN Maeda-Martínez, JA Farías-Sánchez \& PE Saucedo. 2010. Biological performance of the penshell Atrina maura and mussel Mytella strigata under different water flow regimes. Ciencias Marinas 36: 237-248.

Bendschneider K \& RJ Robinson. 1952. A new spectrophotometric method for the determination of nitrite in sea-water. Journal of Marine Resources 11: 87-96.

Bunting W. 2013. Principles of sustainable aquaculture. Promoting social, economic and environmental resilience, 320 pp. CSIRO Pub., Clayton.

Camacho-Mondragón MA, M Arellano-Martinez \& BP Ceballos-Vazquez. 2012. Particular features of gonad maturation and size at first maturity in Atrina maura (Bivalvia: Pinnidae). Scientia Marina 76: 539-548.

Cardoza-Velasco F. 1998. Penshell aquaculture in northwestern Mexico: the effect of tidal height and stocking density on growth of Atrina maura Sowerby, 1835, cultured in bottom plots. Bulletin of Malacological Society of London 31: 11.

Cardoza-Velasco F \& A Maeda-Martínez. 1997. An approach to aquacultural production of the penshell Atrina maura Sowerby, 1835 (Bivalvia: Pinnidae) in northwest Mexico. Journal of Shellfish Research 16: 311.

Coan EV \& P Valentich-Scott. 2012. Bivalve seashells of tropical west America. Marine bivalve mollusks from Baja California to Peru, 1257 pp. Santa Barbara Museum of Natural History, Santa Barbara.

Gallo-García MC, M García-Ulloa, D Godínez-Siordia \& K Rivera-Gómez. 2001. Estudio preliminar sobre el crecimiento y supervivencia del ostión del Pacífico Crassostrea gigas (Thunberg, 1873) en Barra de Navidad, Jalisco, México. Universidad y Ciencia 17: 83-91.

Góngora-Gómez AM, M García-Ulloa, AL DomínguezOrozco \& JA Hernández-Sepúlveda. 2011. Crecimiento del callo de hacha Atrina maura (Sowerby, 1835) (Bivalvia: Pinnidae) cultivado a diferentes densidades. Avances en Investigación Agropecuaria 15: 79-94.

Góngora-Gomez AM, M Garcia-Ulloa, AL DominguezOrozco, JA Hernandez-Sepulveda \& A Nolasco-Orta. 2012. Desarrollo científico y biotecnológico para el cultivo comercial del callo de hacha (Atrina maura) en el Estado de Sinaloa, Mexico, 118 pp, Mundilibros, Tlaxcala.

Hernández SJ. 2006. Crecimiento y sobrevivencia del ostión del Pacífico Crassostrea gigas (Thunberg, 1851) en el estero La Piedra, Guasave, Sinaloa, durante el ciclo de cultivo otoñoprimavera. Tesis de Licenciatura, Instituto Tecnológico de Los Mochis, Los Mochis, Sinaloa, 103 pp. 
Jeffrey SW \& GF Humphrey. 1975. New spectrophotometric equation for determining chlorophyll $\mathrm{a}, \mathrm{b}, \mathrm{c}_{1}$ and $\mathrm{c}_{2}$. Biochemical and Physiology 167: 194-204.

Keen AM. 1971. Sea shells of Tropical West America, 347 pp. Stanford University Press, Stanford.

Langton RN, KC Haines \& RE Lyon. 1977. Ammonianitrogen production by the bivalve mollusc Tapes japonica and its recovery by the red seaweed Hypnea musciformis in a tropical mariculture system. Helgoländer Wissenchaftliche Meeresuntersuchungen 30: 217-229.

Leal-Soto S, R Barraza-Guardado, R Castro-Longoria, J Chávez-Villalba \& F Hoyos-Cháirez. 2011. Cultivation of pen shells: An example with Atrina maura in Northwestern Mexico. Journal of the World Aquaculture Society 42: 789-800.

Leyva-Valencia L, AN Maeda-Martínez, MT Sicard, I Roldan \& M Robles-Mungaray. 2001. Halotolerance, upper thermotolerance, and optimum temperature for growth of the penshell Atrina maura (Sowerby, 1835) (Bivalvia: Pinnidae). Journal of Shellfish Research 20: 49-54.

Lora-Vilchis MC, E Ruiz-Velasco-Cruz, T ReynosoGranados \& D Voltolina. 2004. Evaluation of five microalgae diets for juvenile pen shell Atrina maura. Journal of the World Aquaculture Society 35: 232-236.

Massó-Rojas JA, JA Vélez-Barajas, MC Fajardo-León \& J Singh-Cabanillas. 1996. Principales recursos malacológicos de Baja California Sur. En: Pesquerías relevantes de México, pp. 127-131. Instituto Nacional de la Pesca, Secretaría del Medio Ambiente, Recursos Naturales y Pesca, México.

Mendo T, V Koch, M Wolff, F Sínsel \& C Ruíz-Verdugo. 2011. Feasibility of intertidal bottom culture of the penshell Atrina maura in Bahía Magdalena, Baja California Sur, Mexico. Aquaculture 314: 252-260.

Menzel W. 1991. Estuarine and marine bivalve mollusk culture, 360 pp. CRC Press, Boca Raton.

Miranda-Baeza M. 1994. Cultivo experimental de callo de hacha Atrina maura (Pelecypoda: Pinnidae), en la laguna de Agiabampo, Sonora. Tesis de Licenciatura, Centro de Estudios Superiores de Sonora, Unidad Académica Navojoa, Sonora, $79 \mathrm{pp}$.

Miranda-Baeza A. 1995. El callo de hacha, un molusco con gran potencial en acuacultura. Panorama Acuícola 1: 12-13.

Murphy J \& JP Riley. 1962. A modified single solution method for the determination of phosphates in natural waters. Analytica Chimica Acta 27: 31-36.

Newell RC. 1976. Adaptation to environment: Essays on the physiology of marine animals, $539 \mathrm{pp}$. Butterworth, London.
Robles M, SJ Serrano \& FJ Hoyos. 1996. Cultivo de larvas y producción de semilla de callo de hacha (Atrina maura, Sowerby, 1835) (Mollusca: Pinnidae), en el CREMES de Bahía Kino, Sonora, México (O-024). En: XI Simposio Internacional de Biología Marina, pp. 1-31. La Paz, Baja California Sur.

Robles-Mungaray M. 2004. Desarrollo de la biotecnología para la producción de semilla en laboratorio, diploide y triploide, de callo de hacha Atrina maura (Sowerby, 1835). Tesis de Maestría, Universidad Autónoma de Baja California Sur, Baja California Sur, 66 pp.

Rodríguez-Jaramillo C, AN Maeda-Martínez, ME Valdez, T Reynoso-Granados, P Monsalvo-Spencer, D PradoAncona, F Cardoza-Velasco, M Robles-Mungaray \& MT Sicard. 2001. The effect of temperature on the reproductive maturity of the penshell Atrina maura (Sowerby, 1835) (Bivalvia: Pinnidae). Journal of Shellfish Research 20: 39-47.

Rupp GS, GJ Parsons, RJ Thompson \& MM De Bem. 2005. Influence of environmental factors, season and size at deployment on growth and retrieval of postlarval lion's paw scallop Nodipecten nodosus (Linnaeus 1758) from a subtropical environment. Aquaculture 243: 195-216.

SARH. 1979. Atlas geológico de uso de suelo. Mapa de tipos de vegetación y su distribución, $450 \mathrm{pp}$. Secretaría de Agricultura y Recursos Hidráulicos, SARH, México.

Serrano-Casillas G. 2004. Crecimiento y supervivencia de la almeja catarina Argopecten ventricosus (Sowerby 11, 1842), bajo condiciones de cultivo en suspensión y fondo en Bahía Concepción, Baja California Sur, México. Tesis de Maestría, Universidad Autónoma de Baja California Sur, Baja California Sur, 164 pp.

Serrano-Guzmán SJ. 1997. Influencia de la temperatura en el crecimiento de juveniles de moluscos bivalvos nativos del Pacífico Mexicano. III Congreso Latinoamericano de Malacología (III CLAMA) y VI Reunión Nacional de Malacología y Conquiología (VI RENEMAC), pp. 1-5. Ensenada, Baja California.

Serrano-Gúzman SJ. 2003. Análisis prospectivo de las relaciones morfométricas de Pinna rugosa Sowerby, 1835 (Bivalvia: Pinnidae) en Corralero-Atolengo, Oaxaca, México. Ciencia y Mar 22: 31-39.

Shinn NB. 1941. A colorimetric method for the determination of nitrite. Industrial and Engineering Chemical Analytical Edition 13: 33-35.

Sibaja WG. 1985. Dimensiones de la concha del mejillón Mytella strigata Hanley (Bivalvia: Mytilidae), de la playa de Lepanto, Puntarenas, Costa Rica. Revista de Biología Tropical 33: 59-50. 
Singh-Cabanillas J \& E Michel-Guerrero. 2002. Aspectos biológicos del callo de hacha, Pinna rugosa (Sowerby, 1835), en Bahía Concepción, B.C.S., Memorias del Primer Foro Científico de Pesca Ribereña, pp. 1-2. Instituto Politécnico Nacional-Centro Regional de Investigaciones Pesqueras, La Paz.

Solórzano L. 1969. Determination of ammonia in natural water by the phenol-hypochlorite method. Limnology and Oceanography 14: 799-801.

Strickland J \& T Parsons. 1972. A practical handbook of seawater analysis. Fisheries Research Board of Canada, Bulletin 167: 1-310.
Wallace JC \& TG Reinsnes. 1985. The significance of various environmental parameters for growth of the Iceland scallop, Chlamys islandica (Pectinidae), in hanging culture. Aquaculture 44: 229-242.

Wootton RF. 1991. Ecology of teleost fishes, 404 pp. Fish and Fisheries. Series Y. Chapman \& Hall, London.

Wu RSS \& PKS Shin. 1998. Transplant experiments on growth and mortality of the fan mussel Pinna bicolor. Aquaculture 163:47-62.

Zar JH. 1996. Biostatistical analysis, 593 pp. Prentice-Hall, Englewood Cliffs. 